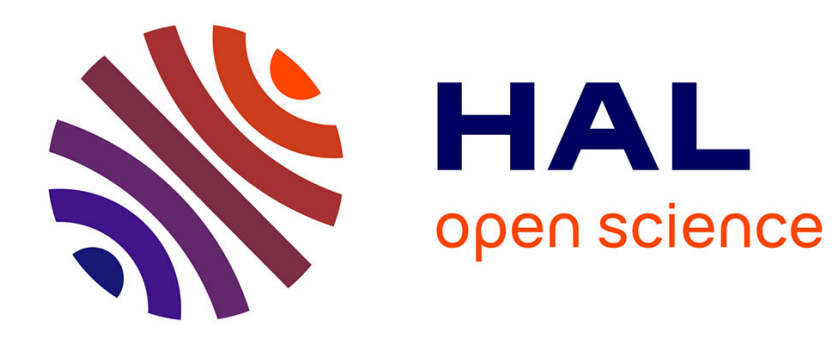

\title{
Pre-peak deformation of model granular materials: a DEM study \\ Jean-Noël Roux
}

\section{To cite this version:}

Jean-Noël Roux. Pre-peak deformation of model granular materials: a DEM study. 3rd International Symposium on Geomechanics from Micro to Macro, Sep 2014, Cambridge, United Kingdom. pp.49-54. hal-01704074

\section{HAL Id: hal-01704074 \\ https://hal.science/hal-01704074}

Submitted on 8 Feb 2018

HAL is a multi-disciplinary open access archive for the deposit and dissemination of scientific research documents, whether they are published or not. The documents may come from teaching and research institutions in France or abroad, or from public or private research centers.
L'archive ouverte pluridisciplinaire HAL, est destinée au dépôt et à la diffusion de documents scientifiques de niveau recherche, publiés ou non, émanant des établissements d'enseignement et de recherche français ou étrangers, des laboratoires publics ou privés. 


\title{
Pre-peak deformation of model granular materials: a DEM study
}

\author{
J.-N. Roux \\ Université Paris Est, Laboratoire Navier \\ Cité Descartes, Champs-sur-Marne, France
}

\begin{abstract}
Initial states of granular assemblies, which determine the response to quasi-statically applied deviator stresses in mechanical tests, are usually classified according to their density and structural anisotropy. Discrete simulations (DEM) of model granular materials (spherical bead packs) reveal however that dense assemblies with isotropic fabric can be prepared with very different coordination numbers, ranging from 4 to 6 under low confining stress. When subsequently subjected to triaxial compression tests equally dense systems differing by their coordination number have the same peak strength, but strongly differ in their pre-peak stressstrain response. Well-coordinated systems have contact networks that tend to resist throughout large deviator intervals, and exhibit a stiff response, with a global stiffness scaling with the contact stiffness (type I strains). Poorly coordinated ones essentially deform in a series of microscopic instabilities in which contact networks get continuously broken and repaired (type II strains). The distinction between strains of types I and II (not to be confused with elastic and plastic strains) has important consequences in terms of sensitivities of macroscopic response to micromechanical features and propensity to develop localization instabilities. The coordination number, which determines to a large extent the type (I or II) of the response, can be related to initial elastic moduli. These observations call for changes in the practice of DEM approaches and refinements in the classification of internal states and stress-strain behaviour of granular materials. Some experimental confrontations are possible.
\end{abstract}

\section{INTRODUCTION}

Discrete element methods (Radjaï \& Dubois 2011, O'Sullivan 2011) have become widespread numerical simulation tools in the investigation of the microscopic, grain-level origins of the macroscopic mechanical properties of granular materials. The essential features of the drained behaviour of sands, as classically probed in triaxial compression tests, are thus reproduced by simulations of model materials such as spherical bead assemblies (Thornton 2000, Suiker \& Fleck 2004). In particular, the deviator stress maximum and the dilatant behaviour of dense samples, the monotonic deviator increase with axial strain and the contractant behaviour of loose samples, as well as the critical state approached for large strain whatever the initial density, are retrieved (Thornton 2000, Radjai $\&$ Roux 2004). The influence of intergranular (contact) friction was assessed (Lemaître et al. 2009), and, beyond the simplest model materials, that of rolling resistance at contacts (Estrada et al. 2008), wide polydispersities (Voivret et al. 2009), non-spherical grain shapes, either smooth (Antony \& Kuhn 2004) or angular (Azéma et al. 2007), have also been investigated. Most studies, however, focus on maximum de- viator stress or on critical state properties, and classify initial states by their sole density (with possible, yet limited, effects of inherent anisotropy).

The purpose of the present communication is to show that the pre-peak behaviour of dense, isotropic systems, is not only determined by the initial density, or influenced by inherent anisotropy (Lam \& Tatsuoka 1988), but is sensitive to the coordination number, which, according to the sample assembling procedure, may vary independently of the density (Agnolin \& Roux 2007a).

\section{MODEL MATERIAL, INITIAL STATE AND ISOTROPIC COMPRESSION}

The numerical model, with the material properties and the simulation procedure are described in detail by Agnolin \& Roux (2007a). We consider assemblies of elastic and frictional spherical beads of diameter $a$, with elastic-frictional contact forces. The friction coefficient is $\mu=0.3$. Contact elasticity abides by Hertz's law for the normal force (Johnson 1985), relating normal force $F_{N}$, to deflection $h$, for a material 
of Young modulus $E$ and Poisson ratio $\nu$, as

$F_{N}=\frac{\tilde{E} \sqrt{a}}{3} h^{3 / 2}$, with $\tilde{E}=\frac{E}{1-\nu^{2}}$

or contact stiffness $K_{N}$ to $h$, or to $F_{N}$, as

$K_{N}=\frac{d F_{N}}{d h}=\frac{\tilde{E} \sqrt{a}}{2} h^{1 / 2}=\frac{1}{2}(3 a)^{1 / 3} \tilde{E}^{2 / 3} F_{N}^{1 / 3}$.

A simplified model is implemented for MindlinDeresiewicz laws (Agnolin \& Roux 2007a) combining tangential elasticity and friction in an energetically consistent way [Elata and Berryman 1996]. Dense numerical samples are assembled under isotropic pressure $P$ in cuboidal cells periodic in all three directions, with appropriate dynamics such that the cell dimensions $L_{x}, L_{y}, L_{z}$ may adapt to prescribed stress levels $\sigma_{x x}, \sigma_{y y}, \sigma_{z z}$. Starting from loose configurations with no contact, all three principal stress values are set to $P$, and the cell is compressed until equilibrium is reached with intergranular forces balancing the prescribed stresses. Particles have the elastic properties of glass (Young modulus $E=70 \mathrm{GPa}$, Poisson ratio $\nu=0.3$ ). Under pressure $P$, a dimensionless stiffness number is defined as

$\kappa=\left(\frac{\tilde{E}}{P}\right)^{2 / 3}$,

so that the typical contact deflection $h$ in contacts, due to the Hertz law (1), satisfies

$\frac{h}{a} \propto \kappa^{-1}$.

The results are based on 5 statistically similar samples of 4000 particles for each preparation procedure. As explained by Agnolin \& Roux (2007a), dense packings are obtained on suppressing the effects of friction in the assembling stage. Setting $\mu=0$ results in the maximum solid fraction $\Phi=0.64$ ("random close packing") of disordered sphere assemblies, and coordination number $z^{*}=6$ (excluding a small proportion $x_{0} \simeq 2 \%$ of "rattlers", grains carrying no force). But if the system is strongly vibrated at high $\Phi$, just below the final equilibrium value, and then finally compressed with $\mu=0.3$, a much lower coordination number is obtained, $z *=4.5$ for nearly equal $\Phi$, while $x_{0}$ exceeds $10 \%$. Samples numerically prepared according to these two methods, subsequently referred to as $\mathrm{A}$ and $\mathrm{C}$, under low pressure $P=10 \mathrm{kPa}$ or $\kappa=39000$ ) are then isotropically compressed (with friction) to various confinement levels (up to $1 \mathrm{MPa}$ or $\kappa=1810$ ), before subjecting them to triaxial compression tests in which "axial strain" $\epsilon_{a}=\epsilon_{1}$ is increased while lateral stresses $\sigma_{2}=\sigma_{3}$ are kept constant. Tab. 1 gives values of solid fraction $\Phi$, coordination number $z^{*}$ (for non-rattler grains) and rattler fraction $x_{0}$ in states $\mathrm{A}$ and $\mathrm{C}$ under isotropic pressure. For reference, we also included similar data on initial state $\mathrm{B}$, prepared by direct compression like $\mathrm{A}$, but with a small friction coefficient $\mu=0.02$, and on initial state $\mathrm{D}$, assembled with the final friction coefficient value $\mu=0.3$. It should be remarked that B is less dense than $\mathrm{C}$, but better coordinated. Under growing pressure, density and coordination numbers increase, but moderately so in the pressure range, up to $1 \mathrm{MPa}$, for which simulation results are presented below. From $10 \mathrm{kPa}(\kappa=39000)$ to $1 \mathrm{MPa}(\kappa=1810), z^{*}$ raises to about 6.2 in A samples, to about 5.1 in $\mathrm{C}$ ones (and $x_{0}$ decreases to about $6 \%$ ), while $\Phi$ increases by less than 0.02 in all cases (Agnolin \& Roux 2007b).

\section{STRESS-STRAIN CURVES AND EFFECT OF CONFINING STRESS}

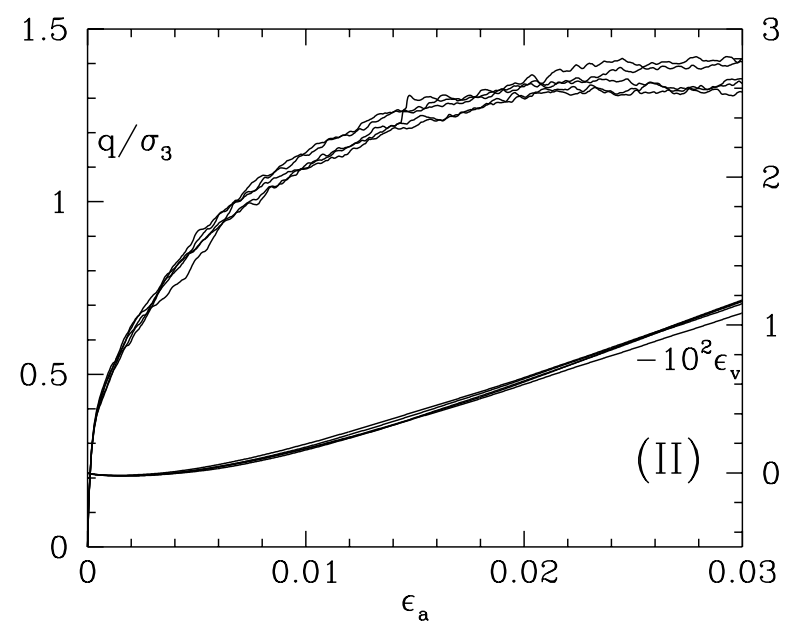

Figure 1: Normalized deviator $q / \sigma_{3}$ and volumetric strain $\epsilon_{v}$ vs. axial strain $\epsilon_{a}$ for 5 different $C$-type samples prepared in the same conditions.

We represent deviator stress, normalized by constant lateral stress, $q / \sigma_{3}=\left(\sigma_{1}-\sigma_{3}\right) / \sigma_{3}$, and volumetric strain $\epsilon_{v}$ versus axial strain $\epsilon_{a}$. Fig. 1 checks for reproducibility of the results on comparing different samples prepared in the same conditions. (Other checks, not shown here in figures for lack of space, show that the implemented DEM procedure indeed probes a quasi-static, rate-independent reponse, and that creep effects vanish for small enough strain rateds $\dot{\epsilon}_{a}$ ). Fig. 2 then compares deviator stress, plotted up to its maximum, and volumetric strain curves obtained from initial states $\mathrm{A}$ and $\mathrm{C}$ for lateral stress $\sigma_{3}=100 \mathrm{kPa}$.

As expected, given their nearly identical initial density, both systems share the same maximum deviator value, about $q / \sigma_{3}=1.4$, within statistical uncertainties (see Fig. 1), corresponding to internal friction angle $\varphi \simeq 24^{\circ}$. Volumetric curves also exhibit the same slope (equal dilatancy), with a more pronounced initial contractant regime in system $\mathrm{C}$. But the axial strain corresponding to a given level of internal friction mobilisation is strikingly different, with, e.g., deviator $q=1.1 \sigma_{3}$ being reached for strains about one 
Table 1: Isotropic states, with $\mu=0.3$ ( $\kappa \simeq 39000$ for $\mathrm{A}$ and $\mathrm{C}, \kappa \simeq 181000$ for $\mathrm{B}$ and $\mathrm{D})$, obtained with different assembling procedures.

\begin{tabular}{|l|ccc|}
\hline Procedure & $\Phi$ & $z^{*}$ & $x_{0}(\%)$ \\
\hline $\mathrm{A}\left(\mu_{0}=0.\right)$ & $0.6370 \pm 0.0002$ & $6.074 \pm 0.0015$ & $1.3 \pm 0.2$ \\
$\mathrm{~B}\left(\mu_{0}=0.02\right)$ & $0.6271 \pm 0.0002$ & $5.80 \pm 0.007$ & $1.95 \pm 0.02$ \\
$\mathrm{C}($ vibrated $)$ & $0.635 \pm 0.002$ & $4.56 \pm 0.03$ & $13.3 \pm 0.5$ \\
$\mathrm{D}\left(\mu_{0}=\mu=0.3\right)$ & $0.5923 \pm 0.0006$ & $4.546 \pm 0.009$ & $11.1 \pm 0.4$ \\
\hline
\end{tabular}

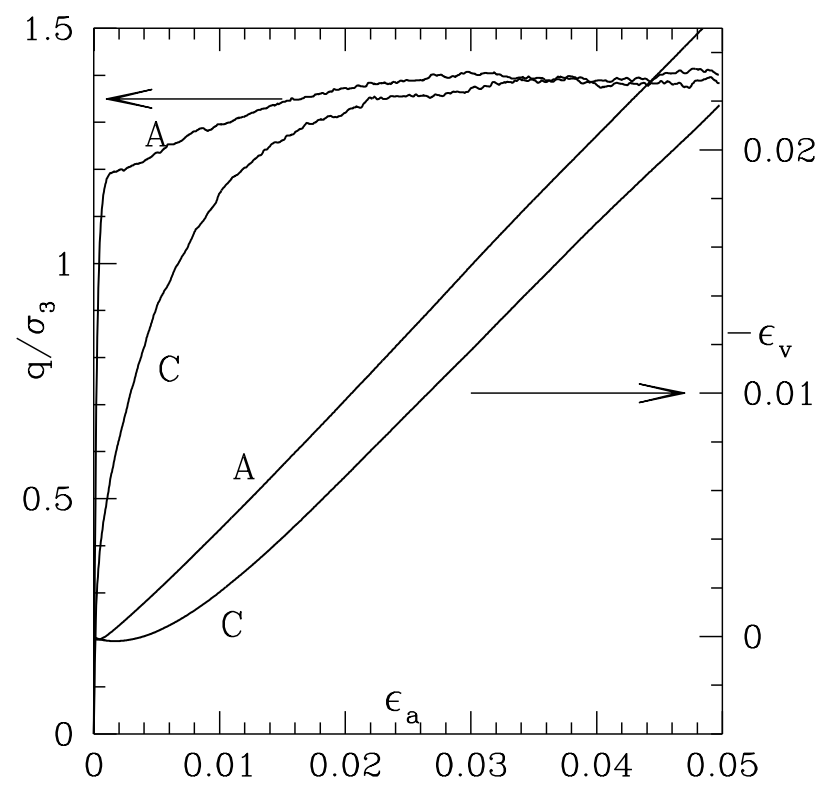

Figure 2: Deviator stress and volumetric strain curves averaged over available A-type and C-type samples.

order of magnitude larger in the $\mathrm{C}$ case $\left(\sim 10^{-2}\right)$ than in systems with initial state $\mathrm{A}\left(\sim 10^{-3}\right)$.

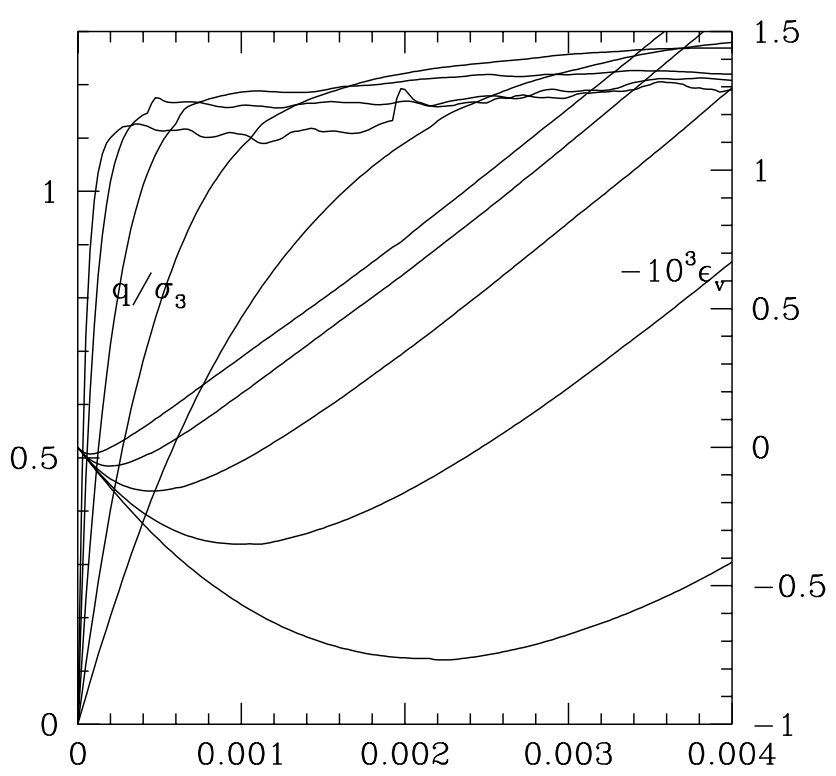

Figure 3: Stress-strain curves for initial pressure $P=\sigma_{3}$, in one A sample, taking 5 different values growing from $10 \mathrm{kPa}$ to $1 \mathrm{MPa}$ by factors of $\sqrt{10}$.

This behaviour is better understood on comparing triaxial tests carried out on one single sample prepared under varying confining pressure. Fig. 3 shows that stress-strain curves on one sample of type A, for $\sigma_{3}$ ranging from $10 \mathrm{kPa}$ to $1 \mathrm{MPa}$, strongly depend on $\sigma_{3}$. The larger the confining stress, the softer the macroscopic response expressed in terms of ratio $q / \sigma_{3}$. To explain such observations, one may note that material deformations, at the microscopic level, i.e., contact deflections, relative to grain diameter $a$, scale as $\kappa^{-1} a$. If macroscopic strains are averages of microscopic ones in the contact regions, then they should scale as $\kappa^{-1} a$ too. This is checked in Fig. 4, which shows that, at least within quite a large initial deviator range, up to about $q=0.9 \sigma_{3}$, stress-strain curves pertaining to different confinement pressures coincide if plotted with stress ratio $q / \sigma_{3}$ and rescaled strains $\kappa \epsilon$.

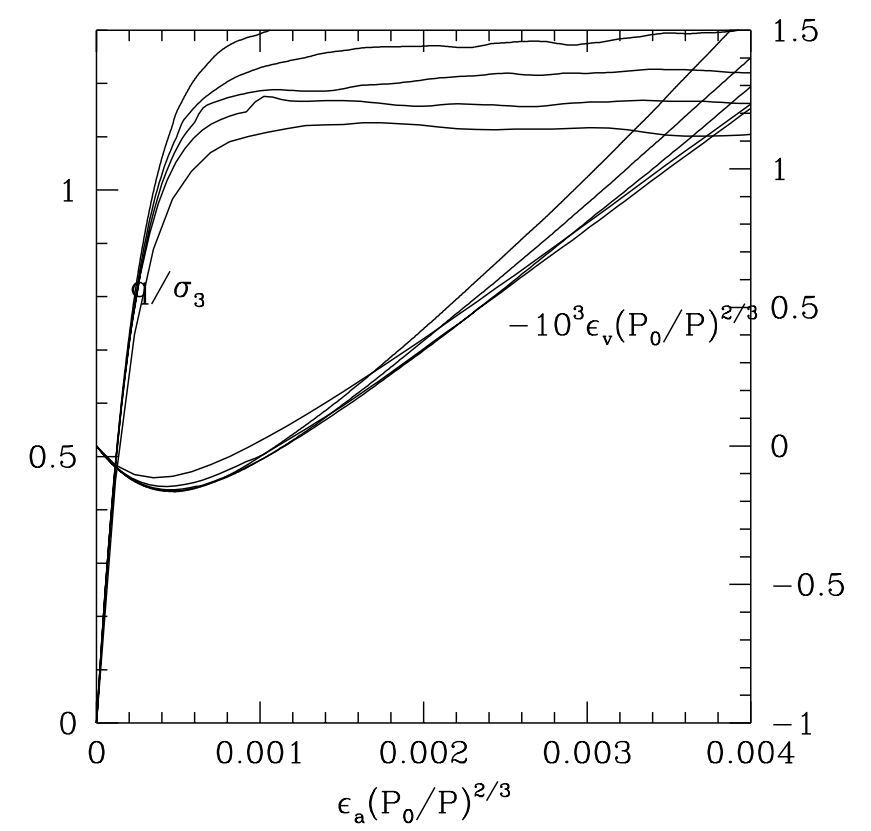

Figure 4: Same as Fig. 3, with rescaled strain coordinates $\left(P_{0}=\right.$ $100 \mathrm{kPa})$.

Fig. 5 and Fig. 6 respectively display stress ratio $q / \sigma_{3}$ and volumetric strain $\epsilon_{v}$ versus axial strain in one C-type sample under the same confining stress values as in Figs. 3-4 (initial pressure $P=\sigma_{3}$ ).

In sharp contrast with the results obtained with Atype systems,stress-strain curves are now reproduced for the different confinement levels without rescaling strains: macroscopic strains do not scale with contact deflections in that case.

Behaviours of A and C-type samples in the prepeak deviator range are characteristic of two different physical origins of macroscopic strains. For a material specimen prepared in state A, strains result from local deformations in the intergranular contact regions - we refer to such strains as type I strains. With C samples, macroscopic strains are of geometric origin, 


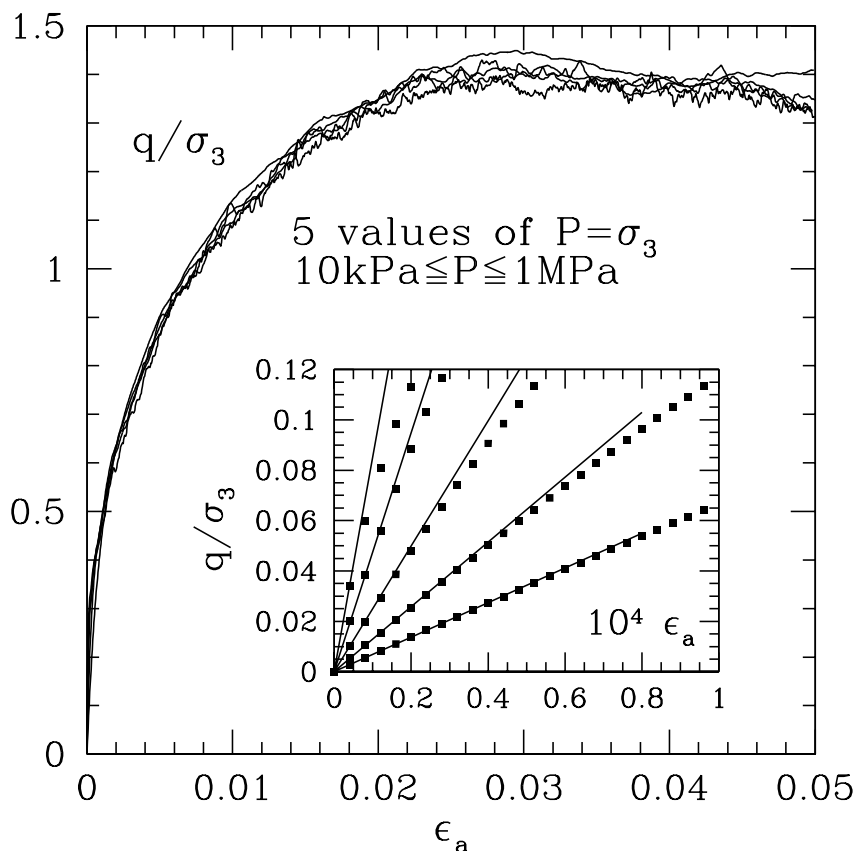

Figure 5: Same as Fig. 3 for normalized deviator stress in one C sample. Inset shows detail of curve near origin (note the blow-up scale).

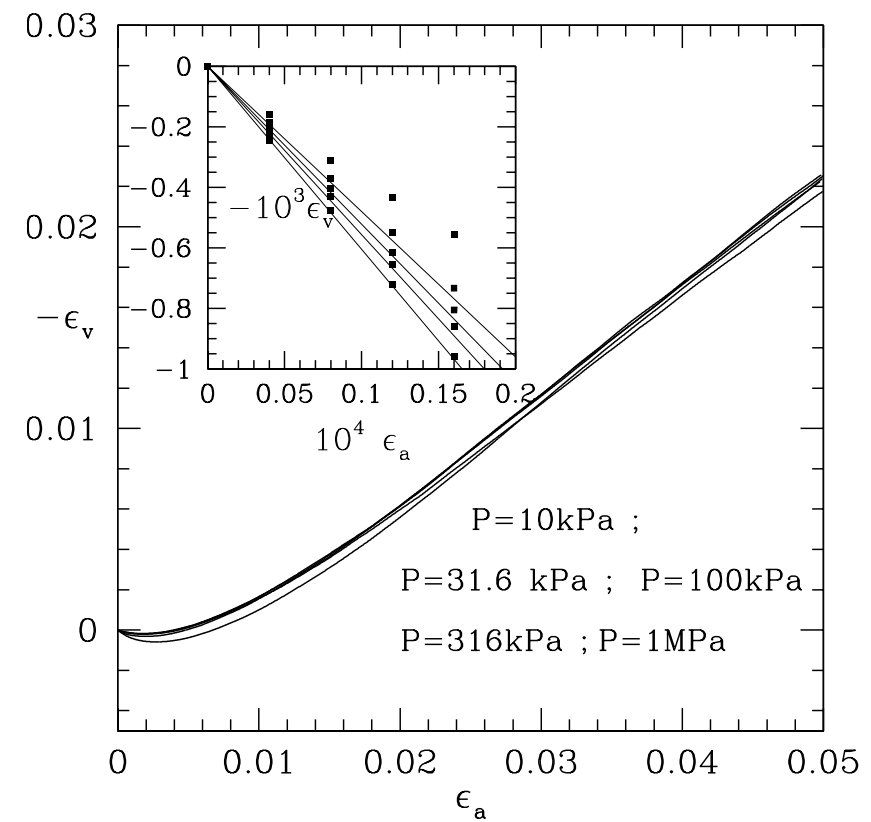

Figure 6: Same as Fig. 3 for volumetric strain in one C sample, the inset magnifying the very small strain region.

stemming from contact network failure and rearrangement (type II strains). Such strains are also present in A samples in a certain deviator range closer to the maximum (say, above $q=\sigma_{3}$ ). One can also observe type I strain response over a significant deviator interval in both systems $\mathrm{A}$ and $\mathrm{C}$ upon unloading (Roux $\&$ Combe 2002), in which both kinds of strains were characterized in some detail with various methods, on two-dimensional numerical simulations of disk assemblies).

An interval of loading parameters in which type I response is observed corresponds to the stability range of a contact network, whence the much larger loading range corresponding to such behaviour in well-coordinated systems such as A. (Actually, a closer scrutiny of results on system $\mathrm{C}$ would reveal a small range of type I response, up to about $q / \sigma_{3}=$ 0.1 ). On unloading, the evolution of tangential contact forces tends to be reversed, thus suppressing full friction mobilisation in many contacts and stabilising the network.

Within a type I strain interval, the system is microscopically stable, each one of its successive configurations can be made arbitrarily close to an equilibrium configuration upon reducing the strain rate. As a consequence, the macroscopic behaviour should be devoid of instability. A strain response of type II, on the other hand, involves microscopic instabilities, as broken contact networks are not repaired until new contacts form, which implies some finite strain interval during which a momentary surge of kinetic energy is observed. This may result in a macroscopic instability which is not, of course, bound to happen. While microscopic stability entails macroscopic stability, microscopic instabilities, which occur with a frequency that increases with the system size (Roux \& Combe 2002) are compatible with well-behaved constitutive laws expressing smooth stress-strain dependencies on the macroscopic scale. The absence of microscopic instability characterising type I strains, or, equivalently, the enduring capability of a given contact network to support varying deviator levels, has been related by (McNamara and Herrmann 2006) to a positive second-order work criterion, expressed in terms of the elastoplastic stifffness matrix of the contact network.

\section{INITIAL ELASTIC PROPERTIES}

In experimental practice, the coordination number is usually not accessible to direct measurements. Yet we now show that elastic moduli, which can be measured (Shibuya et al. 1992, di Benedetto et al. 1999, Kuwano and Jardine 2002), provide indirect information on contact nework coordination. In Figs. 5 and 6 the insets (subplots), with blown-up strain scales, focus on the initial, very small strain response. The straight lines then correspond to the elastic behaviour of the initial contact network, with slope $E^{*}$, the effective Young modulus of the granular system, in Fig. 5, and $-\left(1-2 \nu^{*}\right)$, with $\nu^{*}$ its effective Poisson ratio, in Fig. 6. These moduli are obtained on computing the response of the initial contact network, assuming each contact responds elastically (to see how these moduli are calculated, on building appropriate stiffness matrices, see Agnolin \& Roux (2007c)). We thus observe in simulations that the moduli give the tangent slopes to the stress-strain curves. In a very small range, for strains typically of order $10^{-5}$ (just like in experiments (di Benedetto et al. 1999)), the incremental behaviour about the initial equilibrium state can be regarded as elastic in good approximation(Agnolin \& Roux 2007c). The elastic reange should not be confused with the type I strain regime, as the lat- 


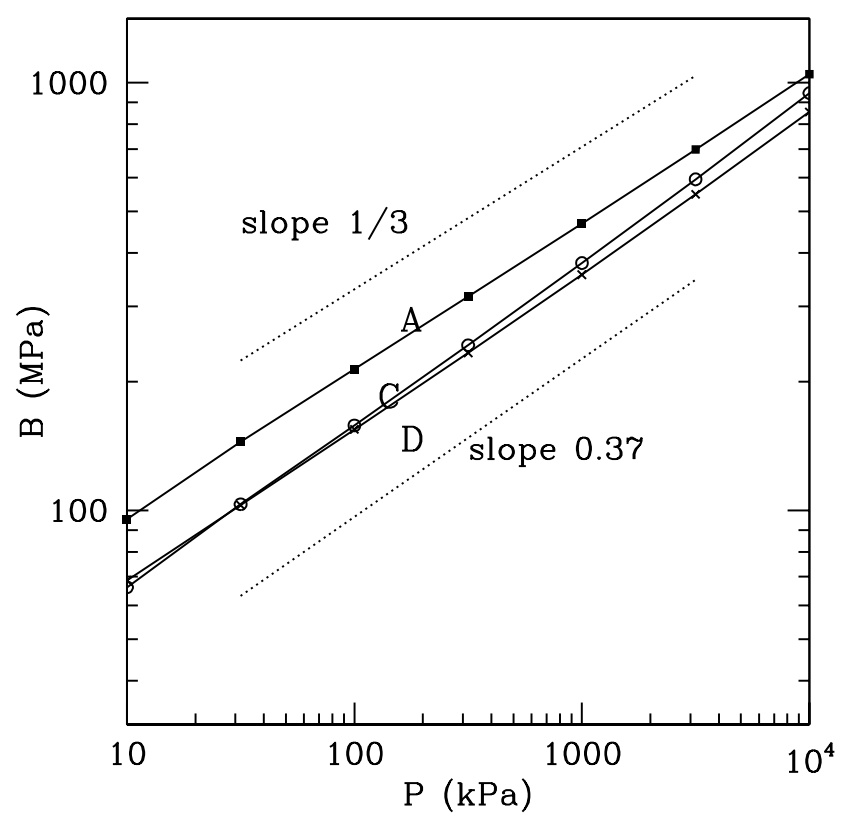

Figure 7: Bulk modulus $B$ vs. pressure $P$, on logarithmic scales, in isotropic compression of samples A, C, D of Tab. 1.

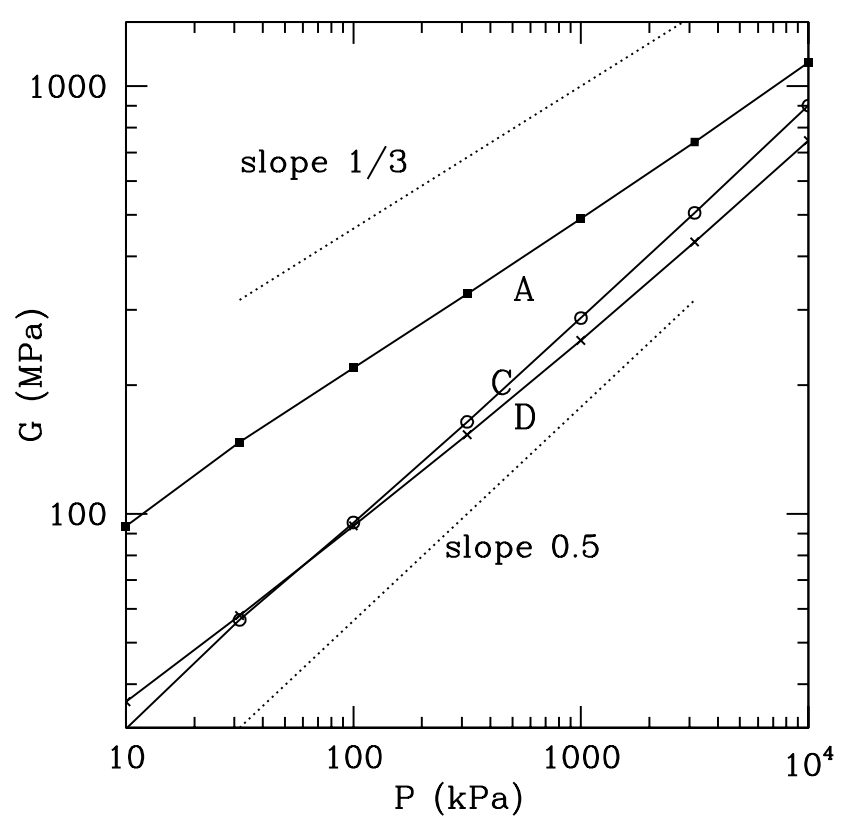

Figure 8: Shear modulus $G$ vs. pressure $P$ in isotropic compression of samples A, C, D of Tab. 1.

ter may obviously extend to much larger stress and strain intervals (Fig. 4). Figs. 7 and 7 respectively plot bulk $(B)$ and shear $(G)$ moduli of all samples of Tab.1, once isotropically compressed, versus pressure $P$. Those graphs make it very clear that moduli are primarily sensitive to coordination number, as $\mathrm{C}$ and $\mathrm{D}$ results are close to each other, despite different densities. Results for state B, not shown in Figs. 7 and 8 are actually close to A ones. Note, also, that those moduli (especially shear modulus $G$ ) of well coordinated and poorly coordinated packings exhibit different pressure dependences - different apparent pressure exponents, see the slopes in the logarithmic plots of Figs. 7 and 8.

More detailed analyses of elastic properties of isotropic spherical particle assemblies are given by Agnolin \& Roux (2007c) (such as a discussion of "anomalous" shear moduli in poorly coordinated isotropic assemblies) along with some experimental comparisons for glass bead systems.

\section{CONCLUSIONS}

Numerical simulations clearly reveal that the initial coordination number in a dense isotropically equilibrated granular material is not determined by the density, and strongly influences the stress-strain response in the pre-peak range. This is related to the ability of well-coordinated contact networks to support large deviator stress increases without failure, whence macroscopic strains of type I. While systems prepared with large coordination numbers exhibit type I strains, i.e., deform because the contacts are deformable, poorly coordinated ones macroscopically deform because contacts networks get continuously broken and repaired. Initial coordination numbers appear to be directly related to elastic moduli.

These observations have important practical and fundamental implications.

On the practical side, these results call for studies of initial state coordination numbers, depending on assembling procedures, which should imply more systematic comparisons between experiments and simulations, preferentially for numerically tractable model materials. It seems that one should eventually refine the classification of initial states in terms of density and inherent fabric anisotropy only, as the coordination number is another source of variability. Results of triaxial tests on sands under varying confining stresses often resemble Figs. 5 and 6 more than Fig. 3, which certainly raises doubts on the relevance of the numerical procedure of compression without friction, most often resorted to in practice because of its simplicity, to produce dense samples.

\section{REFERENCES}

Agnolin, I. \& J.-N. Roux (2007a). Internal states of model isotropic granular packings. I. Assembling process, geometry, and contact networks. Phys. Rev. E 76(6), 061302.

Agnolin, I. \& J.-N. Roux (2007b). Internal states of model isotropic granular packings. II. Compression and pressure cycles. Phys. Rev. E 76(6), 061303.

Agnolin, I. \& J.-N. Roux (2007c). Internal states of model isotropic granular packings. III. Elastic properties. Phys. Rev. E 76(6), 061304.

Antony, S. J. \& M. R. Kuhn (2004). Evolution of force distribution in three-dimensional granular media. Int. J. Solid Struct. 41, 58635870.

Azéma, E., F. Radjaï, R. Peyroux, \& G. Saussine (2007). Force transmission in a packing of pentagonal particles. Phys. Rev. E 76, 011301.

di Benedetto, H., H. Geoffroy, C. Sauzéat, \& B. Cazacliu (1999). Sand behaviour in very small to medium strain domains. In M. Jamiolkowski, R. Lancellotta, and D. Lo Presti (Eds.), Pre-failure Deformation Characteristics of Geomaterials, Rotterdam, pp. 89-96. Balkema. 
Estrada, N., A. Taboada, \& F. Radjaï (2008). Shear strength and force transmission in granular media with rolling resistance. Phys. Rev. E 78, 021301.

Johnson, K. L. (1985). Contact Mechanics. Cambridge University Press.

Kuwano, R. \& R. J. Jardine (2002). On the applicability of crossanisotropic elasticity to granular materials at very small strains. Géotechnique 52, 727-749.

Lam, W.-K. \& F. Tatsuoka (1988). Effects of initial anisotropic fabric and $\sigma_{2}$ on strength and deformation characteristics of soils. Soils and Foundations 28, 89-106.

Lemaître, A., J.-N. Roux, \& F. Chevoir (2009). What do dry granular flows tell us about dense non-brownian suspension rheology? Rheologica Acta 48, 925-942.

McNamara, S. \& H. J. Herrmann (2006). Quasirigidity: some uniqueness issues. Phys. Rev. E 74, 061303.

O'Sullivan, C. (2011). Particulate Discrete Element Modeling: a Geomechanics perspective, Volume 4 of Applied Geotechnics series. Spon Press.

Radjaï, F. \& F. Dubois (2011). Discrete-element modeling of granular materials. Wiley.

Radjaï, F. \& S. Roux (2004). Contact dynamics study of 2d granular media : critical states and relevant internal variables. In H. Hinrichsen and D. E. Wolf (Eds.), The Physics of Granular Media, Berlin, pp. 165-187. Wiley-VCH.

Roux, J.-N. \& G. Combe (2002). Quasistatic rheology and the origins of strain. C. R. Physique 3, 131-140.

Shibuya, S., F. Tatsuoka, S. Teachavorasinskun, X.-J. Kong, F. Abe, Y.-S. Kim, \& C.-S. Park (1992). Elastic deformation properties of geomaterials. Soils and Foundations 32, 26-46.

Suiker, A. S. J. \& N. A. Fleck (2004). Frictional collapse of granular assemblies. ASME Journal of Applied Mechanics 71, 350-358.

Thornton, C. (2000). Numerical simulations of deviatoric shear deformation of granular media. Géotechnique 50, 43-53.

Voivret, C., F. Radjaï, J.-Y. Delenne, \& M. El Youssoufi (2009). Multiscale force networks in highly polydisperse granular media. Phys. Rev. Lett. 102, 178001. 